\title{
SINTOMAS DEPRESSIVOS ENTRE ESTUDANTES DE MEDICINA DE UMA UNIVERSIDADE DA REGIÃO SUL DO TOCANTINS
}

\author{
Depressive Symptoms Among Medical Students Of A University Of The South \\ Tocantins Region
}

Síntomas Depresivos Entre Estudiantes Médicos De Una Universidad De La Región De Tocantinas Del Sur

\section{Vinícius Lopes Marinho*1, Jeann Bruno Ferreira da Silva ${ }^{2}$, Aline Ribeiro Dias ${ }^{3}$, Ana Caroline de Andrade ${ }^{3}$, Lucas Gomes Barcelos ${ }^{3}$, Italo Brito Salera ${ }^{4}$, Bryam Simosen de Oliveira $^{4}$.}

${ }^{1}$ Psicólogo. Doutorando em Ensino pela Universidade do Vale do Taquari, Univates. Mestre em Ciências da Saúde pela Universidade Federal do Tocantins/UFT.

${ }^{2}$ Psicólogo. Doutorando em Desenvolvimento Regional e Mestre em Ciências da Saúde pela Universidade Federal do Tocantins/UFT.

${ }^{3}$ Discente do curso de Psicologia da Universidade de Gurupi/UnirG, Tocantins, Brasil.

${ }^{4}$ Discente do curso de Medicina da Universidade de Gurupi/UnirG, Tocantins, Brasil.

*Correspondência: Universidade de Gurupi/UnirG, Av. Rio de Janeiro $n^{\circ}$ 1585, Centro - 77403-090, GurupiTO, Telefone: (63) 3612-7636, e-mail:vinicius.marinho22@gmail.com.

\section{RESUMO}

Introdução: A Organização Mundial da Saúde estima que a depressão será considerada a doença mais comum nos próximas 3 décadas. Esse agravo já é visível entre o meio universitário, principalmente entre estudantes dos cursos de medicina das universidades brasileiras. Objetivo: Investigar a existência de sintomas depressivos em estudantes de medicina de uma Universidade da região Sul do Tocantins. Material e Métodos: trata-se de pesquisa de campo, de natureza descritiva de cunho quantitativo. Os dados são apresentados por meio de tabelas e gráficos que expressam escores absolutos em forma de percentuais. A coleta ocorreu em dias distintos do ano de 2019 nas próprias salas de aula, em intervalos cedidos pelos docentes. O instrumento utilizado foi o Inventário de Depressão de Beck-BDI. Resultados e discussão: os dados demográficos apontaram que a maioria dos estudantes, $58 \%$, são do sexo feminino e $42 \%$ do sexo masculino. Destes, $69 \%$ não apresentam nenhum tipo de sintomatologia enquanto $31 \%$ apresentam alguma sintomatologia depressiva. $16 \%$ apresenta sintomatologia leve, $12 \%$ moderada e $3 \%$ grave. Considerações finais: A maioria dos entrevistados não apresentou sintomatologia alguma, no entanto há uma distribuição considerável que apresenta os referidos sintomas e este fato evidenciou que há necessidade de intervenção com a referida população.

Palavras-chave: Sintomas depressivos; Estudantes de medicina; Universidade

\section{ABSTRACT}

Introduction: The World Health Organization estimates that depression will be considered the most common disease in the next 3 decades. This grievance is already visible among the university environment, especially among medical students at Brazilian universities. Objective: To investigate the existence of depressive symptoms in medical students at a University in the southern region of Tocantins. Material and Methods: This is a field research, of descriptive nature of quantitative nature. Data are presented through tables and graphs that express absolute scores as percentages. The collection took place on different days of 2019 in the classrooms themselves, at intervals given by 
the teachers. The instrument used was the Beck-BDI Depression Inventory. Results and discussion: Demographic data indicated that the majority of the students, 58\%, are female and $42 \%$ male. Of these, $69 \%$ do not have any symptoms while $31 \%$ have some depressive symptoms. $16 \%$ have mild symptoms, $12 \%$ moderate and $3 \%$ severe. Final Considerations: Most of the interviewees did not present any symptoms, however there is a considerable distribution that presents the referred symptoms and this fact showed that there is a need for intervention with that population.

Keywords: Depressive symptoms; Medical students; University..

\section{RESUMEN}

Introducción: La Organización Mundial de la Salud estima que la depresión se considerará la enfermedad más común en las próximas 3 décadas. Esta queja ya es visible entre el entorno universitario, especialmente entre los estudiantes de medicina de las universidades brasileñas. Objetivo: investigar la existencia de síntomas depresivos en estudiantes de medicina de una universidad en la región sur de Tocantins. Material y métodos: Esta es una investigación de campo, de naturaleza descriptiva de naturaleza cuantitativa. Los datos se presentan a través de tablas y gráficos que expresan puntuaciones absolutas como porcentajes. La recolección tuvo lugar en diferentes días de 2019 en las aulas, a intervalos dados por los maestros. El instrumento utilizado fue el Inventario de depresión Beck-BDI. Resultados y discusión: Los datos demográficos indicaron que la mayoría de los estudiantes, 58\%, son mujeres y 42\% hombres. De estos, el 69\% no tiene ningún síntoma, mientras que el $31 \%$ tiene algunos síntomas depresivos. $16 \%$ tienen síntomas leves, $12 \%$ moderados y 3\% severos. Consideraciones finales: la mayoría de los entrevistados no presentaron ningún síntoma, sin embargo, existe una distribución considerable que presenta los síntomas referidos y este hecho mostró que existe una necesidad de intervención con esa población.

Palabras clave: síntomas depresivos; Estudiantes de medicina; Universidad.

\section{INTRODUÇÃO}

Segundo dados da Organização Mundial da Saúde - OMS, a depressão deve se tornar a doença mais comum do mundo nos próximos 20 anos, acometendo mais pessoas do que o câncer e as doenças cardíacas. A OMS a considera como o quinto maior problema de saúde pública no mundo, com maior incidência nos países pobres, podendo ter relação com elevados níveis de estresse vivenciado por estes (OLIVEIRA, 2013).

A depressão será a doença com os mais elevados custos econômicos e sociais para os governos, devido aos gastos com tratamento para a população e às perdas por afastamento do trabalho. Ainda, a OMS estima que há cerca de 121 milhões de pessoas com depressão no mundo, sendo 17 milhões delas no Brasil (MAGALHÃES, 2011).

Sobre o exposto, cabe aqui uma breve explanação nosológica dos respectivos sintomas. Oliveira (2013) classifica os transtornos depressivos em três grupos, de acordo com a presença dos seguintes sintomas: 1) Depressão menor: dois a quatro sintomas por duas ou mais semanas, incluindo humor deprimido ou anedonia; 2) Distimia: três ou quatro sintomas, incluindo humor deprimido, durante dois anos, no mínimo; 3) Depressão maior: cinco ou mais sintomas por duas semanas ou mais, incluindo humor deprimido ou anedonia, seu tratamento é principalmente medicamentoso, associada ou não à psicoterapia, mas podem ser utilizadas outras alternativas para pacientes com resistência terapêutica, como por exemplo, a estimulação magnética transcraniana (EMT) e a eletroconvulsoterapia.

Estudos epidemiológicos realizados no Brasil têm evidenciado um cenário preocupante acerca dos sintomas depressivos e ansiosos encontrados em um determinado grupo em particular - os estudantes de Medicina - estes apresentam taxas maiores do que a população em geral, podendo ter relação com o curso médico e/ou com características do próprio indivíduo 
em si (BRUCH e CARNEIRO, 2009; TORRES et al., 2012; FURTADO et al.,2013).

Segundo Vasconcelos et al., (2014) os fatores que podem influenciar a prevalência de ansiedade e depressão nos estudantes de medicina são: a elevada carga horária, o grande volume de matérias, o maior contato com pacientes portadores de diversas doenças e prognósticos, a insegurança em relação ao ingresso no mercado de trabalho, cobranças da sociedade e da instituição de ensino superior, além da autocobrança típica do curso.

Oliveira (2013) assinala que o estresse tem sido considerado um fator basilar para $\mathrm{o}$ desencadeamento de sintomas depressivos. Assim, nos últimos anos tem crescido a realização de estudos sobre a temática em questão, especificamente sobre a relação entre o estresse e sintomas depressivos nesta comunidade acadêmica.

O conhecimento dos problemas que afligem os estudantes de Medicina é de essencial importância na execução de planejamento adequado para atender às suas solicitações e também para fornecer subsídios à instituição de ensino, com o intuito de auxiliá-la no aprimoramento da formação discente e de suas relações interpessoais no ambiente universitário.

Diante o exposto, o objetivo deste estudo consistiu em investigar a existência de sintomas depressivos em estudantes de medicina de uma Universidade da região Sul do Tocantins.

\section{MATERIAIS E MÉTODOS}

Trata-se de uma pesquisa de campo com abordagem quantitativo-descritiva. Esse tipo de pesquisa é empregado quando se tem a intenção de obter informações e/ou conhecimentos a respeito de um problema, ou também encontrar novas manifestações, ou a ligação entre eles (LAKATOS \& MARCONI, 2013).
Quantos aos procedimentos de coleta, inicialmente, foi solicitada autorização junto à coordenação do curso de medicina da I.E.S. para realização da pesquisa com os respectivos acadêmicos. Ainda, antes do início da pesquisa. Vale ressaltar que o estudo ocorreu conforme previsto na resolução 466/2012 do Conselho Nacional de Saúde, foi submetido ao Comitê de Ética em Pesquisa com Seres Humanos sob CAAE: 73315717.0.0000.5518 e aprovado conforme parecer $\mathrm{n}^{\circ}$ 3.336.234. Participaram do estudo 363 acadêmicos matriculados entre o primeiro e o oitavo período do curso de medicina.

Os dados foram coletados no entre os meses de abril e outubro de 2019 nas salas de aula em momentos distintos com os acadêmicos do referido curso. Como instrumentos de coleta, foram utilizados um questionário para caracterização do perfil sociodemográfico elaborado pelos autores e o Inventário de Depressão de Beck-BDI que trata-se do instrumento de autoavaliação de depressão mais utilizado em pesquisa e em clínica, tendo sido traduzido para vários idiomas e já foi validada no Brasil. Esta Escala consta de 21 itens relacionados a sintomas como: tristeza, pessimismo, sensação de fracasso, sensação de punição, autodepreciação, ideias suicidas, irritabilidade, retração social, distúrbio do sono, alterações do apetite, entre outros itens identificadores de transtornos de depressão.

Para a análise descritiva dos dados (frequência e porcentagem) foi utilizado o software SPSS Statistical Package for the Social Sciences versão 20.0 para Windows. A apresentação dos resultados dar-seá por meio de tabelas e gráficos.

\section{RESULTADOS E DISCUSSÃO}

Participaram da pesquisa 363 alunos matriculados do primeiro ao oitavo período do curso de medicina. Inicialmente os dados são apresentados 
por meio de tabela (página seguinte) acerca da caracterização do perfil socioeconômico dos participantes, levando em consideração as seguintes variáveis: gênero, faixa etária, estado civil, situação de moradia e atividade remunerada.

Tabela 1. Perfil Socioeconômico dos participantes

\begin{tabular}{l|cc}
\hline Gênero & N & $\mathbf{\%}$ \\
\hline Feminino & 210 & $58 \%$ \\
Masculino & 127 & $42 \%$ \\
\hline Faixa etária & $\mathbf{N}$ & $\mathbf{\%}$ \\
16-25 anos & 281 & $77 \%$ \\
$26-39$ anos & 70 & $20 \%$ \\
$>$ 40 anos & 03 & $1 \%$ \\
Não responderam & 09 & $2 \%$ \\
\hline Estado Civil & $\mathbf{N}$ & $\mathbf{\%}$ \\
Solteiros & 324 & $89 \%$ \\
Casados & 20 & $5 \%$ \\
Divorciados & 1 & $1 \%$ \\
Não responderam & 18 & $5 \%$ \\
\hline Situação de Moradia & $\mathbf{N}$ & $\mathbf{\%}$ \\
Sozinhos & 181 & $50 \%$ \\
Pais & 74 & $20 \%$ \\
Outros familiares & 37 & $10 \%$ \\
Cônjuge & 19 & $6 \%$ \\
Pensionato ou República & 12 & $3 \%$ \\
Outros locais & 28 & $8 \%$ \\
Não informaram & 12 & $3 \%$ \\
\hline Atividade Remunerada & $\mathbf{N}$ & $\mathbf{\%}$ \\
Não trabalham & 312 & $82 \%$ \\
Trabalho remunerado & 49 & $13 \%$ \\
Outros (bolsas) & 6 & $2 \%$ \\
\hline & & \\
\hline
\end{tabular}

Fonte: dados da pesquisa.
Dos estudantes participantes deste estudo 210 (58\%) eram do sexo feminino e 127 (42\%) masculino. Em se tratando da faixa etária $281(77 \%)$ tinham entre 16 e 25 anos, 70 (20\%) entre 26 e 39 anos, 03 (1\%) acima de 40 anos e 9 (2\%) não responderam ao item no questionário. Em relação ao estado civil 324 (89\%) se declararam solteiros, 20(5\%) casados, 1 (1\%) divorciado e 18 (5\%) não informaram no questionário. Do número total de alunos 181 (50\%) moram sozinhos, 74 (20\%) moram com os pais, $37(10 \%)$ moram com outros familiares, 19 (6\%) moram com o conjugue, $12(3 \%)$ moram em pensionato ou republica, $28(8 \%)$ declaram morar em outros locais não mencionados no questionário e 12 (3\%) não informaram.

Tabela 2. Perfil segundo tipo de tratamento realizado

\begin{tabular}{lcc}
\hline \multicolumn{1}{c}{ VARIÁVEIS } & N & \% \\
\hline JA FEZ TRATAMENTO & & \\
PSICOLÓGICO & & \\
Sim & 100 & 28 \\
Não & 196 & 54 \\
Em andamento & 26 & 7 \\
Não informado & 41 & 11 \\
& & \\
JÁ FEZ TRATAMENTO & & \\
PSIQUIÁTRICO & & \\
Sim & 85 & 23 \\
Não & 221 & 61 \\
Em andamento & 39 & 11 \\
Não informado & 18 & 5 \\
\hline
\end{tabular}

Fonte: dados da pesquisa.

A maior parte dos estudantes (82\%) declararam não possuir nenhum tipo de atividade remunerada, dedicando-se integralmente ao curso, 49 (13\%) declararam possuir trabalho remunerado, outros $6(2 \%)$ declararam possuir atividade acadêmica tais como PET, bolsa de estágio, pesquisa, monitoria etc.

$\mathrm{Na}$ Universidade em questão à qual o estudo foi realizado, assim como as demais do país, o curso 
de medicina é de turno integral e nesse caso, há períodos em que a grade curricular sobressai 14 (quatorze) disciplinas por semestre. Fato este que, por si, exprime o tempo disponível para realização de outras atividades.

O presente estudo não objetivou correlacionar as variáveis sociodemográficas com a existência de sintomas depressivos, visto que em estudos como os de Leão (2018) e Bühre (2019) as variáveis sexo, faixa etária, estado civil, religião, renda, com quem reside, ter plano de saúde, motivo da escolha do curso, forma de pagamento do curso, se fuma, bebe ou se há preocupação com o futuro não apresentaram associações significativas com a presença de depressão.

Também foi componente do questionário o inquérito acerca de estarem ou não em tratamento psicológico e psiquiátrico. A tabela abaixo expressa os dados do inquérito.

Ao serem questionados sobre realização de acompanhamento/tratamento psicológico, 100 (28\%) declararam ter realizado acompanhamento, sendo que $26(7 \%)$ em andamento. Em relação ao tratamento psiquiátrico $85(23 \%)$ afirmaram a realização desse tipo de acompanhamento e 39 (11\%) informaram que o mesmo se encontra em andamento. Sobre tal achado, Serr (2015) constatou em seu estudo que dentre 671 estudantes $86,8 \%$ nunca fizeram tratamento psiquiátrico, $68 \%$ nunca fizeram psicoterapia.

$\mathrm{O}$ interesse pelos aspectos psicológicos dos estudantes de medicina e do médico é milenar. Hipócrates (460-377 a.C.) já chamava a atenção para o risco do médico tornar-se onipotente: "o sábio é aquele que procura aprender; quem acredita que a tudo conhece é ignorante". Fazendo referência a característica do médico e daquele que aspira um dia sê-lo, de acreditar ser um semideus. Contudo foi apenas no século XX que tais aspectos passaram a ser estudados de forma ordenada. No Brasil, desde a década de 50, a saúde mental dos estudantes de medicina tem sido tema de estudos (REZENDE, et al., 2008).

Ainda acerca de tal achado, Serr (2015) constatou em seu estudo que dentre 671 estudantes $86,8 \%$ nunca fizeram tratamento psiquiátrico, $68 \%$ nunca fizeram psicoterapia, pode-se inferir que a inexistência desse tipo de tratamento/acompanhamento pode agravar ao longo do tempo até mesmos sintomas mais leves de depressão.

Sobre a prevalência de sintomas depressivos, os dados obtidos após aplicação e correção do inventário de depressão de Beck evidenciaram que apenas $31 \%$ dos participantes possuem algum tipo de sintoma depressivo e $69 \%$ sem nenhum tipo de sintomatologia.

Gráfico 1. Prevalência de sintomas depressivos

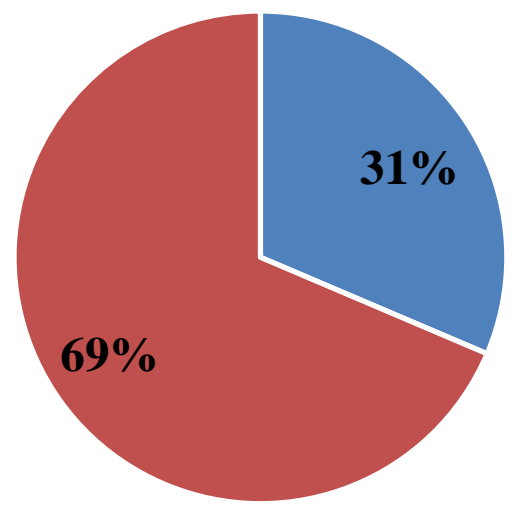

\section{- Sim $\square$ Não}

Fonte: dados da pesquisa.

Os dados deste trabalho foram comparados com outros estudos com o mesmo instrumento, obtendo-se valores semelhantes. Todos mostraram a prevalência da depressão entre os estudantes de medicina mesmo em níveis mais leves. 
Tabalipa (2015) ao estimar a prevalência, gravidade e fatores associados de ansiedade e transtornos depressivos entre estudantes de medicina constatou $32,8 \%$ de depressão na amostra estudada. Ao estimar a prevalência e os fatores associados à depressão e ansiedade entre estudantes universitários da área da saúde de um Centro Universitário no Nordeste do Brasil, Leão (2018) também por meio do Inventário de Depressão de Beck (BDI) e Inventário de Ansiedade de Beck (BAI) com 476 válidos, de 76 alunos do curso de Medicina, constatou que 25,9\% têm depressão. Ao Identificar a frequência e os fatores associados à ocorrência de depressão em estudantes de medicina da Universidade Federal do Amapá, Oliveira (2016) constatou que dos 188 participantes, 45,7\% apresentaram sintomas depressivos, um índice maior ao do presente estudo.

Sobre a classificação nosológica acerca da sintomatologia depressiva, os dados obtidos após aplicação e correção do inventário de depressão de Beck evidenciaram que $69 \%$ da amostra não possui nenhum sintoma depressivo, $16 \%$ apresentaram sintomas leves, $12 \%$ apresentaram sintomas moderados e $3 \%$ sintomas graves. Vide gráfico abaixo.

Gráfico 2. Escore de sintomatologia depressiva nos estudantes de medicina

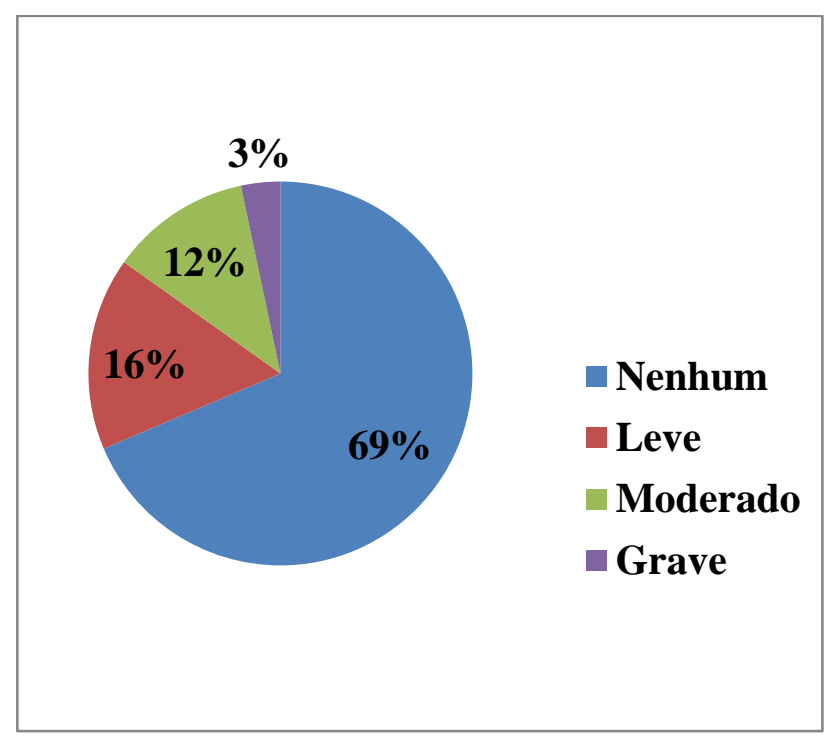

Fonte: dados da pesquisa.

Dentre os 400 acadêmicos avaliados no estudo de Rezende (2008) a prevalência de sintomas depressivos foi de $79 \%$, sendo $29 \%$ com grau leve; $31 \%$ moderado e $19,25 \%$ grave. Já no estudo de Oliveira (2016) dos 188 participantes, 45,7\% apresentaram sintomas depressivos. Ainda em relação a severidade dos sintomas depressivos Tabalipa (2015) constatou que a prevalência foi de $32,8 \%$, sendo $28,2 \%$ leve, $4,2 \%$ moderada e $0,4 \%$ severa.

\section{CONCLUSÃO}

O curso de Medicina, em particular, expõe os estudantes que optam por seguir a carreira médica, a variadas situações de estresse: o curso é o mais longo de todos os cursos universitários, com duração mínima de 6 (seis) anos; a carga horária semestral é bastante extenuante; há uma fadiga extrema pelas horas dedicadas aos estudos pré-avaliações, sendo as noites de sono com frequência substituídas por noites de estudos, para muitos destes futuros médicos. Além disso, há uma auto cobrança para corresponder às próprias expectativas, a de familiares e da sociedade.

Por outro lado, o presente estudo mostrou que os alunos de Medicina da Universidade de Gurupi apresentaram uma prevalência de sintomas depressivos tal qual a encontrada em outros estudos, sendo a taxa de depressão não alterada significativamente, com percentual de $31 \%$.

Em relação ao perfil dos estudantes houve a prevalência do sexo feminino, a faixa etária esteve entre 16 e 25 anos, onde a maior parte são solteiros morando sozinhos e não desenvolvendo nenhum tipo de atividade remunerada, ou seja, dedicando-se integralmente aos estudos. 
Ainda que os escores de sintomas depressivos encontrados nesta pesquisa foram considerados baixos, não se pode negligenciar tais constatações, visto que escores baixos hoje podem evoluir para índices elevados no futuro caso não exista algum tipo de intervenção.

Desta forma o presente estudo sugere a criação de espaços para que os estudantes, não somente de um único curso possam compartilhar anseios, angustias e preocupações presentes no percurso de sua formação profissional, contribuindo assim para a redução de eventos estressores, melhorando assim a qualidade de vida dessa parcela da população.

Todos os autores declararam não haver qualquer potencial conflito de interesses referente a este artigo.

\section{REFERÊNCIAS}

BÜHRER, Bruna Elisa, et al. Análise da Qualidade e Estilo de Vida entre Acadêmicos de Medicina de uma Instituição do Norte do Paraná. Rev. bras. educ. méd, p. $39-46,201$

BRUCH TP, CARNEIRO EA, Jornada LK. Presença de sintomas psiquiátricos em estudantes de medicina de Universidade do sul do Brasil. Arquivos Catarinenses de Medicina, vol.38, n.4, 2009. Disponível em: http://www.acm.org.br/revista/pdf/artigos/770.pdf. Acesso em: 19 dez 2019.

DE REZENDE, C. H. A., et al. Prevalência de sintomas depressivos entre estudantes de medicina da Universidade Federal de Uberlândia. Rev Bras Educ Med, p. 315-323. Uberlândia, Minas Gerais. 2008

DE OLIVEIRA, Gabriella Santos, et al. Prevalência e fatores associados à depressão em estudantes de medicina da Universidade Federal do Amapá. Revista de Medicina e Saúde de Brasília. 2017.

FURTADO ES, FALCONE E M, CLARK C. Avaliação do estresse e das habilidades sociais na experiência acadêmica de estudantes de medicina de uma Universidade do Rio de Janeiro. Interação em Psicologia, v. 7, n. 2. p. 43-51, 2003. Disponível em: http://ojs.c3sl.ufpr.br/ojs2/index.php/psicologia/articl e/view/3222/2584 et al. Acesso em 19 dez 2019.

LEÃO, Andrea Mendes, et al. Prevalência e Fatores Associados à Depressão e Ansiedade entre Estudantes Universitários da Área da Saúde de um Grande Centro Urbano do Nordeste do Brasil Prevalence and Factors Associated with. Revista Brasileira de Educação Médica, p. 55-65, 2018.

MAGALHÃES CC. P. Depressão a doença do século XXI? Psique Ciência \& Vida, ano VI, n. 69, setembro, São Paulo: Escala; ano VI, n. 69, setembro. 2011.

MARCONI, M. D. A., \& LAKATOS, E. M. Fundamentos de metodologia científica. São Paulo: Atlas; 2013.

OLIVEIRA, Elisângela. Prevalência de sintomas depressivos em estudantes de Medicina da Universidade Federal da. Dissertação de Mestrado. Salvador. 2013.

ORGANIZACIÓN MUNDIAL DE LA SALUD (OMS). Estadísticas sanitarias mundiales 2011. Genebra: Organización Mundial de la Salud. 2011. Disponível em: http://www.who.int/entity/whosis/whostat/ES_WHS2 011_Full.pdf. Acesso em: 02 jun. 2019.

SERRA, Rosana Denobile; DINATO, Sandra Lopes Mattos; CASEIRO, Marcos Montani. Prevalence of depressive and anxiety symptoms in medical students in the city of Santos. Jornal Brasileiro de Psiquiatria, p. 213-220, 2015.

TABALIPA, Fábio de Oliveira, et al. Prevalence of anxiety and depression among medical students. Revista Brasileira de Educação Médica, p. 388-394, 2015.

VASCONCELOS et al. Prevalência de Sintomas de Ansiedade e Depressão em Estudantes de Medicina. Revista Brasileira de Educação Médica. v. 39 p. 135-142. 2015. 